\title{
I nteractive fuzzy programming for stochastic two-level linear programming problems through probability maximization
}

\author{
Masatoshi Sakawa, Takeshi Matsui \\ Faculty of Engineering, Hiroshima University, Higashi-Hiroshima, Japan \\ Correspondence: Masatoshi Sakawa. Address: 1-4-1, Kagamiyama, Higashi-Hiroshima, Japan. Telephone: \\ 86-82-424-7694. E-mail: sakawa@hiroshima-u.ac.jp.
}

Received: June 20, 2012

DOI : 10.5430/air.v2n2p109

Accepted: September 10, 2012

Online Published: March 4, 2013

\begin{abstract}
This paper considers two-level linear programming problems involving random variable coefficients both in objective functions and constraints. Using the concept of chance constraints, under some appropriate assumptions for distribution functions, the original stochastic two-level linear programming problems are transformed into deterministic ones. Taking into account vagueness of judgments of the decision makers, in order to derive a satisfactory solution considering satisfactory balance between both levels, an interactive fuzzy programming method is proposed. The proposed method has an advantage that candidates for a satisfactory solution can be easily obtained through the combined use of the bisection method and the phase one of the simplex method. An illustrative numerical example is provided to demonstrate the feasibility of the proposed method.
\end{abstract}

\section{Key words}

Two-level linear programming problems, Random variables, Chance constraints, Probability maximization, Interactive decision making

\section{I ntroduction}

Decision making problems in decentralized organizations are often formulated as two-level programming problems with a decision maker (DM) at the upper level (DM1) and another DM at the lower level (DM2). Under the assumption that these DMs do not have motivation to cooperate mutually, the Stackelberg solution ${ }^{[1-4]}$ is adopted as a reasonable solution for the situation. However, the above assumption is not always reasonable when we deal with decision making problems in a decentralized firm where top management is DM1 and an operation division of the firm is DM2 because it is supposed that there exists cooperative relationship between them. Namely, top management or an executive board is interested in overall management policy such as long-term corporate growth or market share. In contrast, operation divisions of the firm are concerned with coordination of daily activities. After the top management chooses a strategy in accordance with the overall management policy, each division determines goals which are relevant to the strategy chosen by the top management, and it tries to achieve them. In this way, decision making problems in a decentralized firm are often formulated as two-level programming problems where there is essentially cooperative relationship between DM1 and DM2. 
Lai ${ }^{[5]}$ and Shih et al. ${ }^{[6]}$ proposed solution concepts for two-level linear programming problems or multi-level ones such that decisions of DMs in all levels are sequential and all of the DMs essentially cooperate with each other. In their methods, the DMs identify membership functions of the fuzzy goals for their objective functions, and in particular, the DM at the upper level also specifies those of the fuzzy goals for the decision variables. The DM at the lower level solves a fuzzy programming problem with a constraint with respect to a satisfactory degree of the DM at the upper level. Unfortunately, there is a possibility that their method leads a final solution to an undesirable one because of inconsistency between the fuzzy goals of the objective function and those of the decision variables. In order to overcome the problem in their methods, by eliminating the fuzzy goals for the decision variables, Sakawa et al. have proposed interactive fuzzy programming for two-level or multi-level linear programming problems to obtain a satisfactory solution for DMs ${ }^{[7,8]}$. The subsequent works on two-level or multi-level programming have been developing ${ }^{[9-18]}$.

In actual decision making situations, however, we must often make a decision on the basis of vague information or uncertain data. For such decision making problems involving uncertainty, there exist two typical approaches: probabilitytheoretic approach and fuzzy-theoretic one. Stochastic programming, as an optimization method based on the probability theory, have been developing in various ways ${ }^{[19,20]}$, including two stage problems considered by Dantzig ${ }^{[21]}$ and chance constrained programming proposed by Charnes et al. ${ }^{[22]}$. Especially, for multiobjective stochastic linear programming problems, Stancu-Minasian ${ }^{[23]}$ considered the minimum risk approach, while Leclercq ${ }^{[24]}$ and Teghem Jr. et al. ${ }^{[25]}$ proposed interactive methods.

Fuzzy mathematical programming representing the vagueness in decision making situations by fuzzy concepts have been studied by many researchers ${ }^{[26,27]}$. Fuzzy multiobjective linear programming, first proposed by Zimmermann ${ }^{[28]}$, have been also developed by numerous researchers, and an increasing number of successful applications has been appearing ${ }^{[15,27,29-36]}$.

As a hybrid of the stochastic approach and the fuzzy one, Wang et al. considered mathematical programming problems with fuzzy random variables ${ }^{[37]}$, Liu et al. ${ }^{[38]}$ discussed chance constrained programming involving fuzzy parameters. In particular, Hulsurkar et al. ${ }^{[39]}$ applied fuzzy programming to multiobjective stochastic linear programming problems. Unfortunately, however, in their method, since membership functions for the objective functions are supposed to be aggregated by a minimum operator or a product operator, optimal solutions which sufficiently reflect the DM's preference may not be obtained. To cope with the problem, after reformulating multiobjective stochastic linear programming problems using several models for chance constrained programming, Sakawa et al. ${ }^{[15,40-42]}$ presented an interactive fuzzy satisficing method to derive a satisficing solution for the DM as a generalization of their previous results ${ }^{[27,33,43-45]}$.

Realizing the importance of considering not only the fuzziness but also the randomness of coefficients of objective functions and/or constraints in mathematical programming problems, some researchers developed two-stage or multistage fuzzy stochastic programming ${ }^{[46-48]}$. Unfortunately, however, no study has focused on the simultaneous consideration of two-level decision making situations and fuzzy stochastic programming approaches.

Under these circumstances, in this paper, we deal with two-level linear programming problems with random variable coefficients in both objective functions and constraints. Using the concept of chance constraints, stochastic constraints are transformed into deterministic ones. Following the probability maximization model, the minimization of each stochastic objective function is replaced with the maximization of the probability that each objective function is less than or equal to a certain value. Under some appropriate assumptions for distribution functions, the formulated stochastic two-level linear programming problems are transformed into deterministic ones. By considering the fuzziness of human judgments, we present an interactive fuzzy programming method for deriving a satisfactory solution for the DMs by updating the satisfactory degree of the DM at the upper level with considerations of overall satisfactory balance among both levels. 


\section{Stochastic two-level linear programming problems}

Consider two-level linear programming problems with random variable coefficients formulated as:

$$
\left.\begin{array}{cl}
\operatorname{minimize} & \bar{z}_{1}\left(\boldsymbol{x}_{1}, \boldsymbol{x}_{2}\right)=\overline{\boldsymbol{c}}_{11} \boldsymbol{x}_{1}+\overline{\boldsymbol{c}}_{12} \boldsymbol{x}_{2}+\bar{\alpha}_{1} \\
\text { for DM1 } & \\
\text { minimize } & \bar{z}_{2}\left(\boldsymbol{x}_{1}, \boldsymbol{x}_{2}\right)=\overline{\boldsymbol{c}}_{21} \boldsymbol{x}_{1}+\overline{\boldsymbol{c}}_{22} \boldsymbol{x}_{2}+\bar{\alpha}_{2} \\
\text { for DM2 } & A_{1} \boldsymbol{x}_{1}+A_{2} \boldsymbol{x}_{2} \leq \overline{\boldsymbol{b}} \\
\text { subject to } & \boldsymbol{x}_{1} \geq \mathbf{0}, \boldsymbol{x}_{2} \geq \mathbf{0}
\end{array}\right\}
$$

where $\boldsymbol{x}_{1}$ is an $n_{1}$ dimensional decision variable column vector for the DM at the upper level (DM1), $\boldsymbol{x}_{2}$ is an $n_{2}$ dimensional decision variable column vector for the DM at the lower level (DM2), $\overline{\boldsymbol{c}}_{l j}, l=1,2, j=1,2$ are $n_{j}$ dimensional random variable row vectors. Here, we assume that $\overline{\boldsymbol{c}}_{l j}$ is expressed as $\overline{\boldsymbol{c}}_{l j}=c_{l j}^{1}+\bar{t}_{l} c_{l j}^{2}$ where $\bar{t}_{l}, l=1,2$ are mutually independent random variables with mean $M_{l}$ and their distribution functions $T_{l}(\cdot), l=1,2$ are continuous and strictly increasing, and that $\bar{\alpha}_{l}, l=1,2$ are random variables expressed as $\bar{\alpha}_{l}=\alpha_{l}^{1}+\bar{t}_{l} \alpha_{l}^{2}$. This definition of random variables is one of the simplest randomization modeling of coefficients using dilation and translation of random variables, as discussed by Stancu-Minasian ${ }^{[23]}$ and Katagiri et al. ${ }^{[49,50]}$. In addition, $\bar{b}_{i}, i=1,2, \ldots, m$ are mutually independent random variables whose distribution functions are also assumed to be continuous and strictly increasing.

Stochastic two-level linear programming problems formulated as (1) are often seen in actual decision making situations, e.g., a supply chain planning ${ }^{[11]}$ where the distribution center (DM1) and the production part (DM2) hope to minimize the distribution cost and the production cost respectively under constraints about inventory levels and production levels. Since coefficients of these objective functions and those of the right-hand side of constraints like product demands are often affected by the economic conditions varying at random, they can be regarded as random variables and the supply chain planning is formulated as (1).

Since (1) contains random variable coefficients, solution methods for ordinary deterministic two-level linear programming problems cannot be directly applied. Consequently, in this paper, we consider the constraints involving random variable coefficients in (1) as chance constraints ${ }^{[22]}$ which mean the probability that each constraint is fulfilled must be greater than or equal to a certain probability (satisficing level). Namely, replacing constraints in (1) by chance constraints with satisficing levels $\beta_{i} \in(0,1), i=1,2, \ldots, m$ problem (1) can be transformed as:

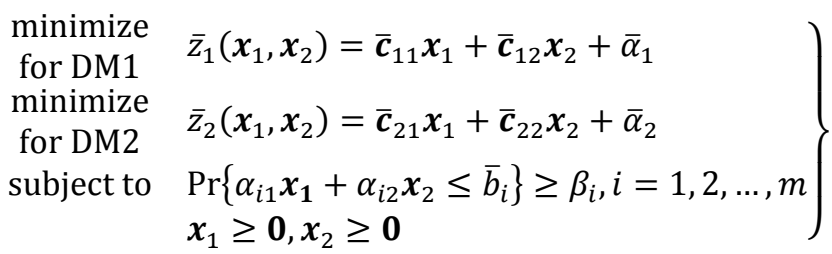

where $a_{i 1}$ and $a_{i 2}$ is the $i$ th row vector of $A_{1}$ and $A_{2}$, and $\bar{b}_{i}$ is the $i$ th element of $\bar{b}$.

Since the distribution function $F_{i}(r)=\operatorname{Pr}\left\{\bar{b}_{i} \leq r\right\}$ of each random variable $\bar{b}_{i}$ is nondecreasing, the $i$ th constraint in (2) can be rewritten as:

$$
\begin{aligned}
\operatorname{Pr}\left\{\alpha_{i 1} \boldsymbol{x}_{1}+\alpha_{i 2} \boldsymbol{x}_{2} \leq \bar{b}_{i}\right\} \geq \beta_{i} & \Leftrightarrow 1-\operatorname{Pr}\left\{\alpha_{i 1} \boldsymbol{x}_{1}+\alpha_{i 2} \boldsymbol{x}_{2} \geq \bar{b}_{i}\right\} \geq \beta_{i} \\
& \Leftrightarrow 1-F_{i}\left(\alpha_{i 1} \boldsymbol{x}_{1}+\alpha_{i 2} \boldsymbol{x}_{2}\right) \geq \beta_{i} \\
& \Leftrightarrow F_{i}\left(\alpha_{i 1} \boldsymbol{x}_{1}+\alpha_{i 2} \boldsymbol{x}_{2}\right) \geq 1-\beta_{i} \\
& \Leftrightarrow \alpha_{i 1} \boldsymbol{x}_{1}+\alpha_{i 2} \boldsymbol{x}_{2} \leq F_{i}^{-1}\left(1-\beta_{i}\right)
\end{aligned}
$$


where $F_{i}^{-1}(\cdot)$ is an inverse function of $F_{i}(\cdot)$.

Letting $\hat{b}_{i}=F_{i}^{-1}\left(1-\beta_{i}\right)$, problem (2) can be rewritten as:

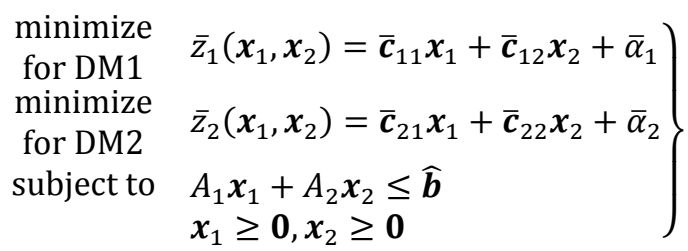

where $\widehat{\boldsymbol{b}}=\left(\hat{b}_{1}, \hat{b}_{2}, \ldots, \hat{b}_{m}\right)^{T}$.

It should be noted here that the constraint of (3) is no longer stochastic but becomes deterministic through the idea of chance constraint.

In addition to the chance constraints, it is now appropriate to consider objective functions with randomness on the basis of some decision making model. As such decision making models, expectation optimization, variance minimization, probability maximization and fractile criterion optimization are typical. For instance, let the objective function represent a profit. If the DM wishes to simply maximize the expected profit without caring about the fluctuation of the profit, the expectation optimization model ${ }^{[51]}$ to optimize the expectation of the objective function is appropriate. On the other hand, if the DM hopes to decrease the fluctuation of the profit as little as possible from the viewpoint of the stability of the profit, the variance minimization model ${ }^{[51]}$ to minimize the variance of the objective function is useful. In contrast to these two types of optimizing approaches, as satisficing approaches, the probability maximization model ${ }^{[51]}$ and the fractile criterion optimization model or Kataoka's model ${ }^{[52]}$ have been proposed. When the DM wants to maximize the probability that the profit is greater than or equal to a certain permissible level, probability maximization model ${ }^{[51]}$ is recommended. In contrast, when the DM wishes to optimize such a permissible level under a given threshold probability with respect to the achieved profit, the fractile criterion optimization model will be appropriate. In this paper, assuming that the DM wants to maximize the probability that the profit is greater than or equal to a certain permissible level for safe management, we adopt the probability maximization model as a decision making model.

In the probability maximization model, the minimization of each objective function $\bar{z}_{l}\left(\boldsymbol{x}_{1}, \boldsymbol{x}_{2}\right)$ in (3) is substituted with the maximization of the probability that $\bar{z}_{l}\left(\boldsymbol{x}_{1}, \boldsymbol{x}_{2}\right)$ is less than or equal to a certain permissible level $h_{l}$ under the chance constraints. Through probability maximization, problem (3) can be rewritten as:

$$
\left.\begin{array}{cl}
\text { maximize } & \operatorname{Pr}\left\{\bar{z}_{1}\left(\boldsymbol{x}_{1}, \boldsymbol{x}_{2}\right) \leq h_{1}\right\} \\
\text { for DM1 } & \\
\text { maximize } & \operatorname{Pr}\left\{\bar{z}_{2}\left(\boldsymbol{x}_{1}, \boldsymbol{x}_{2}\right) \leq h_{2}\right\} \\
\text { for DM2 } & \\
\text { subject to } & A_{1} \boldsymbol{x}_{1}+A_{2} \boldsymbol{x}_{2} \leq \widehat{\boldsymbol{b}} \\
& \boldsymbol{x}_{1} \geq \mathbf{0}, \boldsymbol{x}_{2} \geq \mathbf{0}
\end{array}\right\}
$$

Supposing that $\boldsymbol{c}_{l 1}^{2} \boldsymbol{x}_{1}+\boldsymbol{c}_{l 2}^{2} \boldsymbol{x}_{2}+\alpha_{l}^{2}>0, l=1,2, \ldots, k$ for any feasible solution $\left(\boldsymbol{x}_{1}, \boldsymbol{x}_{2}\right)$ to (4), from the assumption on the distribution function $T_{l}(\cdot)$ of each random variable $\bar{t}_{l}$, we can rewrite objective functions in (4) as follows. 


$$
\begin{aligned}
& \operatorname{Pr}\left\{\bar{z}_{l}\left(\boldsymbol{x}_{1}, \boldsymbol{x}_{2}\right) \leq h_{l}\right\} \\
= & \operatorname{Pr}\left\{\left(\boldsymbol{c}_{l 1}^{1}+\bar{t}_{l} \boldsymbol{c}_{l 2}^{2}\right) \boldsymbol{x}_{1}+\left(\boldsymbol{c}_{l 2}^{1}+\bar{t}_{l} \boldsymbol{c}_{l 2}^{2}\right) \boldsymbol{x}_{2}+\left(\alpha_{l}^{1}+\bar{t}_{l} \alpha_{l}^{2}\right) \leq h_{l}\right\} \\
= & \operatorname{Pr}\left\{\left(\boldsymbol{c}_{l 1}^{2} \boldsymbol{x}_{1}+\boldsymbol{c}_{l 2}^{2} \boldsymbol{x}_{2}+\alpha_{l}^{2}\right) \bar{t}_{l}+\left(\boldsymbol{c}_{l 1}^{1} \boldsymbol{x}_{1}+\boldsymbol{c}_{l 2}^{1} \boldsymbol{x}_{2}+\alpha_{l}^{1}\right) \leq h_{l}\right\} \\
= & \operatorname{Pr}\left\{\bar{t}_{l} \leq \frac{h_{l}-\left(\boldsymbol{c}_{l 1}^{1} \boldsymbol{x}_{1}+c_{l 2}^{1} \boldsymbol{x}_{2}+\alpha_{l}^{1}\right)}{\left(\boldsymbol{c}_{l 1}^{2} \boldsymbol{x}_{1}+\boldsymbol{c}_{l 2}^{2} \boldsymbol{x}_{2}+\alpha_{l}^{2}\right)}\right\} \\
= & T_{l}\left(\frac{h_{l}-\boldsymbol{c}_{l 1}^{1} \boldsymbol{x}_{1}-\boldsymbol{c}_{l 2}^{1} \boldsymbol{x}_{2}-\alpha_{l}^{1}}{\boldsymbol{c}_{l 1}^{2} \boldsymbol{x}_{1}+\boldsymbol{c}_{l 2}^{2} \boldsymbol{x}_{2}+\alpha_{l}^{2}}\right)
\end{aligned}
$$

Hence, (4) can be equivalently transformed into the following deterministic two-level programming problem.

$$
\left.\begin{array}{cl}
\underset{\operatorname{maximize}}{\operatorname{mar} \mathrm{DM} 1} & p_{1}\left(\boldsymbol{x}_{1}, \boldsymbol{x}_{2}\right)=T_{1}\left(\frac{h_{1}-\boldsymbol{c}_{11}^{1} \boldsymbol{x}_{1}-c_{12}^{1} \boldsymbol{x}_{2}-\alpha_{1}^{1}}{\boldsymbol{c}_{11}^{2} \boldsymbol{x}_{1}+c_{12}^{2} x_{2}+\alpha_{1}^{2}}\right) \\
\text { maximize } & p_{2}\left(\boldsymbol{x}_{1}, \boldsymbol{x}_{2}\right)=T_{2}\left(\frac{h_{2}-\boldsymbol{c}_{21}^{1} \boldsymbol{x}_{1}-\boldsymbol{c}_{22}^{1} \boldsymbol{x}_{2}-\alpha_{2}^{1}}{\boldsymbol{c}_{21}^{2} \boldsymbol{x}_{1}+c_{22}^{2} \boldsymbol{x}_{2}+\alpha_{2}^{2}}\right) \\
\text { for DM2 } & A_{1} \boldsymbol{x}_{1}+A_{2} \boldsymbol{x}_{2} \leq \widehat{\boldsymbol{b}} \\
\text { subject to } & \boldsymbol{x}_{1} \geq \mathbf{0}, \boldsymbol{x}_{2} \geq \mathbf{0}
\end{array}\right\}
$$

In this way, through the ideas of chance constraint and probability maximization model, the original stochastic two-level programming problem is transformed into the deterministic two-level programming problem.

\section{I nteractive fuzzy programming}

Considering imprecise natures inherent in human judgments, it is quite natural to assume that the DMs may have fuzzy goals for each of the objective functions in the deterministic two-level linear programming problem (5). To be more specific, in order to consider the imprecise nature of the DMs' judgments for the probabilities $p_{l}\left(\boldsymbol{x}_{1}, \boldsymbol{x}_{2}\right), l=1,2$ in (5), it seems natural to assume that the DMs have fuzzy goals such as " $p_{l}\left(\boldsymbol{x}_{1}, \boldsymbol{x}_{2}\right)$ should be substantially greater than or equal to some specific value.” Then, (5) can be rewritten as:

$$
\left.\begin{array}{cl}
\begin{array}{c}
\operatorname{maximize} \\
\text { for DM1 }
\end{array} & \mu_{1}\left(p_{1}\left(\boldsymbol{x}_{1}, \boldsymbol{x}_{2}\right)\right) \\
\text { maximize } & \mu_{2}\left(p_{2}\left(\boldsymbol{x}_{1}, \boldsymbol{x}_{2}\right)\right) \\
\text { for DM2 } & A_{1} \boldsymbol{x}_{1}+A_{2} \boldsymbol{x}_{2} \leq \widehat{\boldsymbol{b}} \\
\text { subject to } & \boldsymbol{x}_{1} \geq \mathbf{0}, \boldsymbol{x}_{2} \geq \mathbf{0}
\end{array}\right\}
$$

where $\mu_{l}(\cdot)$ is a membership function to quantify a fuzzy goal for the $l$ th objective function in (5) and it is assumed to be nondecreasing. Although the membership function does not always need to be linear, for the sake of simplicity, we adopt a linear membership function. To be more specific, if the DM feels that $p_{l}\left(\boldsymbol{x}_{1}, \boldsymbol{x}_{2}\right)$ should be greater than or equal to at least $p_{l, 0}$ and $p_{l}\left(\boldsymbol{x}_{1}, \boldsymbol{x}_{2}\right) \geq p_{l, 1}\left(>p_{l, 0}\right)$ is satisfactory, the linear membership function $\mu_{l}\left(p_{l}\left(\boldsymbol{x}_{1}, \boldsymbol{x}_{2}\right)\right)$ is defined as:

$$
\mu_{l}\left(p_{l}\left(\boldsymbol{x}_{1}, \boldsymbol{x}_{2}\right)\right)=\left\{\begin{array}{cl}
0 & , p_{l}\left(\boldsymbol{x}_{1}, \boldsymbol{x}_{2}\right)<p_{l, 0} \\
\frac{p_{l}\left(\boldsymbol{x}_{1}, \boldsymbol{x}_{2}\right)-p_{l, 0}}{p_{l, 1}-p_{l, 0}} & , p_{l, 0} \leq p_{l}\left(\boldsymbol{x}_{1}, \boldsymbol{x}_{2}\right) \leq p_{l, 1} \\
1 & , p_{l}\left(\boldsymbol{x}_{1}, \boldsymbol{x}_{2}\right)>p_{l, 1}
\end{array}\right.
$$

and it is depicted in Figure 1. 


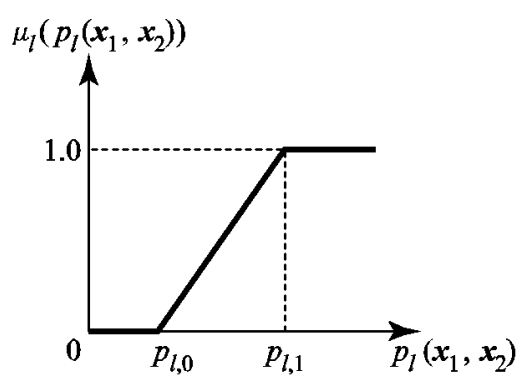

Figure 1. Linear membership function.

Zimmermann ${ }^{[28]}$ suggested a method for assessing the parameter values of the linear membership function. In his method, the parameter values $p_{l, 1}, l=1,2$ are determined as

$$
\begin{aligned}
& p_{1,1}=p_{1, \text { max }}=p_{1}\left(\boldsymbol{x}_{1, \text { max }}^{1}, \boldsymbol{x}_{2, \text { max }}^{1}\right)=\max _{\left(\boldsymbol{x}_{1}^{T}, \boldsymbol{x}_{2}^{T}\right)^{T} \in X} p_{1}\left(\boldsymbol{x}_{1}, \boldsymbol{x}_{2}\right) \\
& p_{2,1}=p_{2, \text { max }}=p_{2}\left(\boldsymbol{x}_{1, \text { max }}^{2}, \boldsymbol{x}_{2, \text { max }}^{2}\right)=\max _{\left(\boldsymbol{x}_{1}^{T}, \boldsymbol{x}_{2}^{T}\right)^{T} \in X} p_{2}\left(\boldsymbol{x}_{1}, \boldsymbol{x}_{2}\right)
\end{aligned}
$$

and the parameter values $p_{l, 0}, l=1,2$ are specified as

$$
\begin{aligned}
& p_{1,0}=p_{1}\left(\boldsymbol{x}_{1, \text { max }}^{2}, \boldsymbol{x}_{2, \text { max }}^{2}\right) \\
& p_{2,0}=p_{2}\left(\boldsymbol{x}_{1, \text { max }}^{1}, \boldsymbol{x}_{2, \text { max }}^{1}\right)
\end{aligned}
$$

where $\left(\boldsymbol{x}_{1, \min }^{l}, \boldsymbol{x}_{2 \text {,min }}^{l}\right)$ is an optimal solution to the following problem

$$
\left.\begin{array}{ll}
\text { maximize } & p_{l}\left(\boldsymbol{x}_{1}, \boldsymbol{x}_{2}\right)=T_{l}\left(\frac{h_{l}-c_{11}^{1} \boldsymbol{x}_{1}-c_{l 2}^{1} \boldsymbol{x}_{2}-\alpha_{l}^{1}}{c_{l 1}^{2} \boldsymbol{x}_{1}+c_{l 2}^{2} \boldsymbol{x}_{2}+\alpha_{l}^{2}}\right) \\
\text { subject to } & A_{1} \boldsymbol{x}_{1}+A_{2} \boldsymbol{x}_{2} \leq \widehat{\boldsymbol{b}} \\
& \boldsymbol{x}_{1} \geq \mathbf{0}, \boldsymbol{x}_{2} \geq \mathbf{0}
\end{array}\right\}
$$

From the monotonicity of the distribution function $T_{l}(\cdot)$, problem (8) is equivalent to:

$$
\left.\begin{array}{ll}
\text { maximize } & \frac{h_{l}-c_{l 1}^{1} x_{1}-c_{l 2}^{1} x_{2}-\alpha_{l}^{1}}{c_{l 1}^{2} x_{1}+c_{l 2}^{2} x_{2}+\alpha_{l}^{2}} \\
\text { subject to } & A_{1} \boldsymbol{x}_{1}+A_{2} \boldsymbol{x}_{2} \leq \widehat{\boldsymbol{b}} \\
& \boldsymbol{x}_{1} \geq \mathbf{0}, \boldsymbol{x}_{2} \geq \mathbf{0}
\end{array}\right\}
$$

Using the variable transformation method by Charnes and Cooper ${ }^{[54]}: s^{l}=1 /\left(\boldsymbol{c}_{l 1}^{2} \boldsymbol{x}_{1}+\boldsymbol{c}_{l 2}^{2} \boldsymbol{x}_{2}+\alpha_{l}^{2}\right), y_{j}=s^{l} \cdot \boldsymbol{x}_{j}, s^{l}>0, l$ $=1,2, j=1,2$, problem (9) is equivalently transformed as:

$$
\left.\begin{array}{cl}
\text { maximize } & -\boldsymbol{c}_{l 1}^{1} \boldsymbol{y}_{1}-\boldsymbol{c}_{l 2}^{1} \boldsymbol{y}_{2}-\left(\alpha_{l}^{1}-h_{l}\right) \cdot s^{l} \\
\text { subject to } & A_{1} \boldsymbol{y}_{1}+A_{2} \boldsymbol{y}_{2}-\widehat{\boldsymbol{b}} \cdot s^{l} \leq 0 \\
& \boldsymbol{c}_{l 1}^{2} \boldsymbol{y}_{1}+\boldsymbol{c}_{l 2}^{2} \boldsymbol{y}_{2}+\alpha_{l}^{2} \cdot s^{l}=1 \\
& \boldsymbol{x}_{1} \geq \mathbf{0}, \boldsymbol{x}_{2} \geq \mathbf{0}
\end{array}\right\}
$$


Since (10) is a linear programming problem, it can be easily solved by the simplex method of linear programming.

To derive an overall satisfactory solution to the membership function maximization problem (6), we first find the maximizing decision of the fuzzy decision proposed by Bellman and Zadeh ${ }^{[53]}$. Namely, the following problem is solved for obtaining a solution which maximizes the smaller degree of satisfaction between those of the two DMs:

$$
\left.\begin{array}{ll}
\operatorname{maximize} & \min \left\{\begin{array}{l}
\left\{\mu_{l}\left(p_{l}\left(\boldsymbol{x}_{1}, \boldsymbol{x}_{2}\right)\right)\right\} \\
\text { subject to }
\end{array}\right. \\
& A_{1} \boldsymbol{x}_{1}+A_{2} \boldsymbol{x}_{2} \leq \widehat{\boldsymbol{b}} \\
\boldsymbol{x}_{1} \geq \mathbf{0}, \boldsymbol{x}_{2} \geq \mathbf{0}
\end{array}\right\}
$$

or equivalently,

$$
\left.\begin{array}{ll}
\operatorname{maximize} & v \\
\text { subject to } & \mu_{1}\left(p_{1}\left(\boldsymbol{x}_{1}, \boldsymbol{x}_{2}\right)\right) \geq v \\
& \mu_{2}\left(p_{2}\left(\boldsymbol{x}_{1}, \boldsymbol{x}_{2}\right)\right) \geq v \\
& A_{1} \boldsymbol{x}_{1}+A_{2} \boldsymbol{x}_{2} \leq \widehat{\boldsymbol{b}} \\
& \boldsymbol{x}_{1} \geq \mathbf{0}, \boldsymbol{x}_{2} \geq \mathbf{0}
\end{array}\right\}
$$

Since $\mu_{l}(\cdot), l=1,2$ are nondecreasing, (12) can be converted as:

$$
\left.\begin{array}{ll}
\operatorname{maximize} & v \\
\text { subject to } & p_{1}\left(\boldsymbol{x}_{1}, \boldsymbol{x}_{2}\right) \geq \mu_{1}^{*}(v) \\
& p_{2}\left(\boldsymbol{x}_{1}, \boldsymbol{x}_{2}\right) \geq \mu_{2}^{*}(v) \\
& A_{1} \boldsymbol{x}_{1}+A_{2} \boldsymbol{x}_{2} \leq \widehat{\boldsymbol{b}} \\
& \boldsymbol{x}_{1} \geq \mathbf{0}, \boldsymbol{x}_{2} \geq \mathbf{0}
\end{array}\right\}
$$

where $\mu_{l}^{*}(\cdot)$ is a pseudo-inverse function of $\mu_{l}(\cdot)$ defined by $\mu_{l}^{*}(r)=\inf \left\{\boldsymbol{y} \mid \mu_{l}(\boldsymbol{y}) \geq r\right\}$. Since

$$
p_{l}\left(\boldsymbol{x}_{1}, \boldsymbol{x}_{2}\right)=T_{l}\left(\frac{h_{l}-\boldsymbol{c}_{l 1}^{1} \boldsymbol{x}_{1}-\boldsymbol{c}_{l 2}^{1} \boldsymbol{x}_{2}-\alpha_{l}^{1}}{\boldsymbol{c}_{l 1}^{2} \boldsymbol{x}_{1}+\boldsymbol{c}_{l 2}^{2} \boldsymbol{x}_{2}+\alpha_{l}^{2}}\right)
$$

and distribution functions $T_{l}(\cdot)$ are assumed to be continuous and strictly increasing, problem (13) is equivalently transformed as:

$$
\left.\begin{array}{ll}
\text { maximize } & v \\
\text { subject to } & \frac{h_{1}-c_{11}^{1} x_{1}-c_{12}^{1} x_{2}-\alpha_{1}^{1}}{c_{11}^{2} x_{1}+c_{12}^{2} x_{2}+\alpha_{1}^{2}} \geq T_{1}^{-1}\left(\mu_{1}^{*}(v)\right) \\
& \frac{h_{2}-c_{21}^{1} x_{1}-c_{22}^{1} x_{2}-\alpha_{2}^{1}}{c_{21}^{2} x_{1}+c_{22}^{2} x_{2}+\alpha_{2}^{2}} \geq T_{2}^{-1}\left(\mu_{2}^{*}(v)\right) \\
& A_{1} \boldsymbol{x}_{1}+A_{2} \boldsymbol{x}_{2} \leq \widehat{\boldsymbol{b}} \\
& \boldsymbol{x}_{1} \geq \mathbf{0}, \boldsymbol{x}_{2} \geq \mathbf{0}
\end{array}\right\}
$$

where $T_{l}^{-1}(\cdot)$ is an inverse function of $T_{l}(\cdot)$.

Obtaining the optimal value of $v$ to (14) is equivalent to finding the maximum of $v$ so that the set of feasible solutions to (14) is not empty. Noting that the constraints of (14) are linear when $v$ is fixed, we can easily find the maximum of $v$ through the combined use of the bisection method and the phase one of the simplex method. 


\section{The combined use of the bisection method and the phase one of the simplex method}

Step 1: Set $r:=0$ and $v:=0$. Test whether the set of feasible solutions to (14) for $v=0$ is empty or not using the phase one of the simplex method. Let $v_{\text {feasible }}:=v$ and go to step 2 .

Step 2: Set $v:=1$. Test whether the set of feasible solutions to (14) for $v=1$ is empty or not using the phase one of the simplex method. If it is not empty, $v=1$ is the optimal value $v^{*}$ to (14) and the algorithm is terminated. Otherwise, the maximum of $v$ so that the set of feasible solutions to (14) is not empty exists between 0 and 1 . Let $v_{\text {infeasible }}:=v$ and go to step 3.

Step 3: Set $v:=\left(v_{\text {feasible }}+v_{\text {infeasible }}\right) / 2, r:=r+1$ and go to step 4 .

Step 4: Test whether the set of feasible solutions to (14) for $v$ determined in step 3 is empty or not, using the phase one of the simplex method. If it is not empty and (1/2)r $\leq \epsilon$, the current value of $v$ is regarded as the optimal value $v^{*}$ to (14) and the algorithm is terminated. If it is not empty and $(1 / 2) r>\epsilon$, let $v_{\text {feasible }}:=v$ and go to step 3 . On the other hand, if it is empty, let $v_{\text {infeasible }}:=v$ and go to step 3 .

For the optimal value $v^{*}$ obtained in this way, we can determine the corresponding optimal solution $\boldsymbol{x}^{*}$ by solving the following linear programming problem.

$$
\left.\begin{array}{cl}
\text { maximize } & \frac{h_{1}-c_{11}^{1} \boldsymbol{x}_{1}-\boldsymbol{c}_{12}^{1} \boldsymbol{x}_{2}-\alpha_{1}^{1}}{\boldsymbol{c}_{11}^{2} \boldsymbol{x}_{1}+c_{12}^{2} \boldsymbol{x}_{2}+\alpha_{1}^{2}} \\
\text { subject to } & \frac{h_{2}-\boldsymbol{c}_{21}^{1} \boldsymbol{x}_{1}-\boldsymbol{c}_{22}^{1} \boldsymbol{x}_{2}-\alpha_{2}^{1}}{\boldsymbol{c}_{21}^{2} \boldsymbol{x}_{1}+\boldsymbol{c}_{22}^{2} \boldsymbol{x}_{2}+\alpha_{2}^{2}} \geq T_{2}^{-1}\left(\mu_{2}^{*}(v)\right) \\
& A_{1} \boldsymbol{x}_{1}+A_{2} \boldsymbol{x}_{2} \leq \widehat{\boldsymbol{b}} \\
& \boldsymbol{x}_{1} \geq \mathbf{0}, \boldsymbol{x}_{2} \geq \mathbf{0}
\end{array}\right\}
$$

Letting $\tau=T_{2}^{-1}\left(\mu_{2}^{*}\left(v^{*}\right)\right)$ and using the variable transformation method by Charnes and Cooper ${ }^{[54]}$, problem (15) can be transformed into the following linear programming problem:

$$
\left.\begin{array}{ll}
\text { maximize } & -\boldsymbol{c}_{11}^{1} \boldsymbol{y}_{1}-\boldsymbol{c}_{12}^{1} \boldsymbol{y}_{2}-\left(\alpha_{1}^{1}-h_{1}\right) \cdot s \\
\text { subject to } & \tau \cdot\left(\boldsymbol{c}_{21}^{2} \boldsymbol{y}_{1}+\boldsymbol{c}_{22}^{2} \boldsymbol{y}_{2}+\alpha_{2}^{2} \cdot s\right)+\boldsymbol{c}_{21}^{1} \boldsymbol{y}_{1}-\boldsymbol{c}_{22}^{1} \boldsymbol{y}_{2}+\left(\alpha_{2}^{1}-h_{2}\right) \cdot s \leq 0 \\
& A_{1} \boldsymbol{y}_{1}+A_{2} \boldsymbol{y}_{2}-\widehat{\boldsymbol{b}} \cdot s \leq 0 \\
& \boldsymbol{c}_{11}^{2} \boldsymbol{y}_{1}+\boldsymbol{c}_{12}^{2} \boldsymbol{y}_{2}+\alpha_{1}^{2} \cdot s=1 \\
& \boldsymbol{x}_{1} \geq \mathbf{0}, \boldsymbol{x}_{2} \geq \mathbf{0}
\end{array}\right\}
$$

From the optimal solution $\left(\boldsymbol{y}_{1}^{*}, \boldsymbol{y}_{2}^{*}, s^{*}\right)$ to (16), we can obtain the optimal solution $\left(\boldsymbol{x}_{1}^{*}, \boldsymbol{x}_{2}^{*}\right)$ to (11) which maximizes the smaller satisfactory degree between those of both DMs.

If DM1 is satisfied with the optimal solution $\left(\boldsymbol{x}_{1}^{*}, \boldsymbol{x}_{2}^{*}\right)$ to (11), it follows that the optimal solution $\left(\boldsymbol{x}_{1}^{*}, \boldsymbol{x}_{2}^{*}\right)$ becomes a satisfactory solution; however, DM1 is not always satisfied with the solution $\left(\boldsymbol{x}_{1}^{*}, \boldsymbol{x}_{2}^{*}\right)$. It is quite natural to assume that DM1 specifies the minimal satisfactory level $\widehat{\delta} \in(0,1)$ for the membership function $\mu_{1}\left(p_{1}\left(\boldsymbol{x}_{1}, \boldsymbol{x}_{2}\right)\right)$ subjectively.

Consequently, if DM1 is not satisfied with the solution $\left(\boldsymbol{x}_{1}^{*}, \boldsymbol{x}_{2}^{*}\right)$ to problem (11), the following problem is formulated:

$$
\left.\begin{array}{ll}
\text { maximize } & \mu_{2}\left(p_{2}\left(\boldsymbol{x}_{1}, \boldsymbol{x}_{2}\right)\right) \\
\text { subject to } & \mu_{1}\left(p_{1}\left(\boldsymbol{x}_{1}, \boldsymbol{x}_{2}\right)\right) \geq \hat{\delta} \\
& A_{1} \boldsymbol{x}_{1}+A_{2} \boldsymbol{x}_{2} \leq \widehat{\boldsymbol{b}} \\
& \boldsymbol{x}_{1} \geq \mathbf{0}, \boldsymbol{x}_{2} \geq \mathbf{0}
\end{array}\right\}
$$


equivalently,

$$
\left.\begin{array}{ll}
\text { maximize } & \frac{h_{2}-c_{21}^{1} x_{1}-c_{22}^{1} x_{2}-\alpha_{2}^{1}}{c_{21}^{2} x_{1}+c_{22}^{2} x_{2}+\alpha_{2}^{2}} \\
\text { subject to } & \frac{h_{1}-c_{11}^{1} x_{1}-c_{12}^{1} x_{2}-\alpha_{1}^{1}}{c_{11}^{2} x_{1}+c_{12}^{2} x_{2}+\alpha_{1}^{2}} \geq T_{1}^{-1}\left(\mu_{1}^{*}(\hat{\delta})\right) \\
& A_{1} \boldsymbol{x}_{1}+A_{2} \boldsymbol{x}_{2} \leq \widehat{\boldsymbol{b}} \\
& \boldsymbol{x}_{1} \geq \mathbf{0}, \boldsymbol{x}_{2} \geq \mathbf{0}
\end{array}\right\}
$$

where DM2's membership function $\mu_{2}\left(p_{2}\left(\boldsymbol{x}_{1}, \boldsymbol{x}_{2}\right)\right)$ is maximized under the condition that DM1's membership function $\mu_{1}\left(p_{1}\left(\boldsymbol{x}_{1}, \boldsymbol{x}_{2}\right)\right)$ is larger than or equal to the minimal satisfactory level $\widehat{\delta}$ specified by DM1.

Using the variable transformation method by Charnes and Cooper ${ }^{[54]}$, problem (18) can be easily reduced to the following linear programming problem:

$$
\left.\begin{array}{ll}
\text { maximize } & -\boldsymbol{c}_{21}^{1} \boldsymbol{y}_{1}-\boldsymbol{c}_{22}^{1} \boldsymbol{y}_{2}-\left(\alpha_{2}^{1}-h_{2}\right) \cdot s \\
\text { subject to } & \lambda \cdot\left(\boldsymbol{c}_{11}^{2} \boldsymbol{y}_{1}+\boldsymbol{c}_{12}^{2} \boldsymbol{y}_{2}+\alpha_{1}^{2} \cdot s\right)+\boldsymbol{c}_{11}^{1} \boldsymbol{y}_{1}-\boldsymbol{c}_{12}^{1} \boldsymbol{y}_{2}+\left(\alpha_{1}^{1}-h_{1}\right) \cdot s \leq 0 \\
& A_{1} \boldsymbol{y}_{1}+A_{2} \boldsymbol{y}_{2}-\widehat{\boldsymbol{b}} \cdot s \leq 0 \\
& \boldsymbol{c}_{21}^{2} \boldsymbol{y}_{1}+\boldsymbol{c}_{22}^{2} \boldsymbol{y}_{2}+\alpha_{2}^{2} \cdot s=1 \\
& \boldsymbol{x}_{1} \geq \mathbf{0}, \boldsymbol{x}_{2} \geq \mathbf{0}
\end{array}\right\}
$$

where $\lambda=T_{1}^{-1}\left(\mu_{1}^{*}(\widehat{\delta})\right)$.

If there exists an optimal solution $\left(\boldsymbol{x}_{1}^{*}, \boldsymbol{x}_{2}^{*}\right)$ to problem (17), it follows that DM1 obtains a satisfactory solution having a satisfactory degree larger than or equal to the minimal satisfactory level specified by DM1's self. However, the larger the minimal satisfactory level $\hat{\delta}$ is assessed, the smaller the DM2's satisfactory degree becomes when the membership functions of DM1 and DM2 conflict with each other. Consequently, a relative difference between the satisfactory degrees of DM1 and DM2 becomes larger, and it follows that the overall satisfactory balance between both DMs is not appropriate.

In order to take account of the overall satisfactory balance between both DMs, DM1 needs to compromise with DM2 on DM1's own minimal satisfactory level. To do so, the following ratio of the satisfactory degree of DM2 to that of DM1 is helpful:

$$
\Delta=\frac{\mu_{2}\left(p_{2}\left(\boldsymbol{x}_{1}, \boldsymbol{x}_{2}\right)\right)}{\mu_{1}\left(p_{1}\left(\boldsymbol{x}_{1}, \boldsymbol{x}_{2}\right)\right)}
$$

which is originally introduced by Lai ${ }^{[5]}$.

DM1 is guaranteed to have a satisfactory degree larger than or equal to the minimal satisfactory level for the fuzzy goal because the corresponding constraint is involved in problem (17). To take into account the overall satisfactory balance between both DMs, DM1 specifies the lower bound $\Delta_{\min }$ and the upper bound $\Delta_{\max }$ of the ratio $\Delta$, and $\Delta$ is evaluated by verifying whether or not it is in the interval $\left[\Delta_{\min }, \Delta_{\max }\right]$. The condition that the overall satisfactory balance is appropriate is represented by

$$
\Delta \in\left[\Delta_{\min }, \Delta_{\max }\right]
$$

At the iteration $k$, let $\left(\boldsymbol{x}_{1}^{k}, \boldsymbol{x}_{2}^{k}\right), p_{l}^{k}=p_{l}\left(\boldsymbol{x}_{1}^{k}, \boldsymbol{x}_{2}^{k}\right), \mu_{l}\left(p_{l}^{k}\right)$ and $\Delta^{k}=\mu_{2}\left(p_{2}^{k}\right) / \mu_{1}\left(p_{1}^{k}\right)$ denote the current solution, DMl's objective function value, DMl's satisfactory degree and the ratio of satisfactory degrees of the two DMs, respectively. The 
interactive process terminates if the following two conditions are satisfied and DM1 concludes the solution as an overall satisfactory solution.

[Termination conditions of the interactive process]

Condition 1 DM1's satisfactory degree is larger than or equal to the minimal satisfactory level $\widehat{\delta}$ specified by DM1's self, i.e., $\mu_{1}\left(p_{1}^{k}\right) \geq \hat{\delta}$.

Condition 2 The ratio $\Delta^{k}$ of satisfactory degrees lies in the closed interval between the lower and the upper bounds specified by DM1, i.e., $\Delta^{k} \in\left[\Delta_{\min }, \Delta_{\max }\right]$.

Condition 1 ensures the minimal satisfaction to DM1 in the sense of the attainment of the fuzzy goal, and condition 2 is provided in order to keep overall satisfactory balance between both DMs. If these two conditions are not satisfied simultaneously, DM1 needs to update the minimal satisfactory level $\widehat{\delta}$.

The updating procedures are summarized as follows.

[Procedure for updating the minimal satisfactory level $\widehat{\delta}$ ]

Case 1 If condition 1 is not satisfied, then DM1 decreases the minimal satisfactory level $\widehat{\delta}$.

Case 2 If the ratio $\Delta^{k}$ exceeds its upper bound, then DM1 increases the minimal satisfactory level $\widehat{\delta}$. Conversely, if the ratio $\Delta^{k}$ is below its lower bound, then DM1 decreases the minimal satisfactory level $\widehat{\delta}$.

Case 3 Although conditions 1 and 2 are satisfied, if DM1 is not satisfied with the obtained solution and judges that it is desirable to increase the satisfactory degree of DM1 at the expense of the satisfactory degree of DM2, then DM1 increases the minimal satisfactory level $\widehat{\delta}$. Conversely, if DM1 judges that it is desirable to increase the satisfactory degree of DM2 at the expense of the satisfactory degree of DM1, then DM1 decreases the minimal satisfactory level $\widehat{\delta}$.

In particular, if condition 1 is not satisfied, there does not exist any feasible solution for problem (17), and therefore DM1 has to moderate the minimal satisfactory level.

Now we are ready to propose interactive fuzzy programming for deriving a satisfactory solution by updating the satisfactory degree of the DM at the upper level with considerations of overall satisfactory balance among all the levels.

\section{Computational procedure of interactive fuzzy programming}

Step 1: Ask the DM at the upper level, DM1, to subjectively determine satisficing levels $\beta_{i} \in(0,1), i=1,2, \ldots, m$ for constraints in (2). Go to step 2.

Step 2: In order to determine permissible levels $h_{l}, l=1,2$, the following problems are solved to find the minimum and maximum of $\mathrm{E}\left\{\bar{z}_{l}\left(\boldsymbol{x}_{1}, \boldsymbol{x}_{2}\right)\right\}=\left(\boldsymbol{c}_{l 1}^{1}+M_{l} \boldsymbol{c}_{l 1}^{2}\right) \boldsymbol{x}_{1}+\left(\boldsymbol{c}_{l 2}^{1}+M_{l} \boldsymbol{c}_{l 2}^{2}\right) \boldsymbol{x}_{2}+\left(\alpha_{l}^{1}+M_{l} \alpha_{l}^{2}\right)$ for each objective function under the chance constraints with satisficing levels $\beta_{i}, i=1,2, \ldots, m$.

$$
\left.\begin{array}{ll}
\text { minimize } & \left(\boldsymbol{c}_{11}^{1}+M_{1} \boldsymbol{c}_{11}^{2}\right) \boldsymbol{x}_{1}+\left(\boldsymbol{c}_{12}^{1}+M_{1} \boldsymbol{c}_{12}^{2}\right) \boldsymbol{x}_{2}+\left(\alpha_{1}^{1}+M_{1} \alpha_{1}^{2}\right) \\
\text { subject to } & A_{1} \boldsymbol{x}_{1}+A_{2} \boldsymbol{x}_{2} \leq \widehat{\boldsymbol{b}} \\
& \boldsymbol{x}_{1} \geq \mathbf{0}, \boldsymbol{x}_{2} \geq \mathbf{0}
\end{array}\right\}
$$




$$
\left.\begin{array}{ll}
\text { minimize } & \left(\boldsymbol{c}_{21}^{1}+M_{2} \boldsymbol{c}_{21}^{2}\right) \boldsymbol{x}_{1}+\left(\boldsymbol{c}_{22}^{1}+M_{2} \boldsymbol{c}_{22}^{2}\right) \boldsymbol{x}_{2}+\left(\alpha_{2}^{1}+M_{2} \alpha_{2}^{2}\right) \\
\text { subject to } & A_{1} \boldsymbol{x}_{1}+A_{2} \boldsymbol{x}_{2} \leq \widehat{\boldsymbol{b}} \\
& \boldsymbol{x}_{1} \geq \mathbf{0}, \boldsymbol{x}_{2} \geq \mathbf{0}
\end{array}\right\}
$$

If the set of feasible solutions to these problems is empty, the satisficing levels $\beta_{i}, i=1,2, \ldots, m$ must be reassessed and return to step 1. Otherwise, let $z_{l, \min }^{E}$ and $z_{l, \max }^{E}$ be optimal objective function values to (20) and (21). Since (20) and (21) are linear programming problems, they can be easily solved by the simplex method. Ask DM1 to determine permissible levels $h_{l}, l=1,2$ for objective functions in consideration of $z_{l, \min }^{E}$ and $z_{l, \max }^{E}$. Go to step 3.

Step 3: Solve (8) for obtaining optimal solutions $\left(\boldsymbol{x}_{1, \max }^{l}, \boldsymbol{x}_{2, \max }^{l}\right), l=1,2$ and calculate $p_{l, \max }$. Then, identify the linear membership function $\mu_{l}\left(p_{l}\left(\boldsymbol{x}_{1}, \boldsymbol{x}_{2}\right)\right)$ of the fuzzy goal for the corresponding objective function. Go to step 4 .

Step 4: Set $k:=1$. Solve the maximin problem (11) for obtaining an optimal solution which maximizes the smaller degree of satisfaction between those of the two DMs. For the optimal solution $\left(\boldsymbol{x}_{1}^{k}, \boldsymbol{x}_{2}^{k}\right)$ to (11), calculate $p_{l}^{k}=p_{l}\left(\boldsymbol{x}_{1}^{k}, \boldsymbol{x}_{2}^{k}\right)$, $\mu_{l}\left(p_{l}^{k}\right), l=1,2$ and $\Delta^{k}=\mu_{2}\left(p_{2}^{k}\right) / \mu_{1}\left(p_{1}^{k}\right)$. If DM1 is satisfied with the optimal solution to (11), the optimal solution becomes a satisfactory solution and the interaction procedure is terminated. Otherwise, ask DM1 to subjectively set the minimal satisfactory level $\widehat{\delta} \in(0,1)$ for the membership function $\mu_{1}\left(p_{1}\left(\boldsymbol{x}_{1}, \boldsymbol{x}_{2}\right)\right)$. Furthermore, ask DM1 to set the upper bound $\Delta_{\max }$ and the lower bound $\Delta_{\min }$ for $\Delta$. Go to step 5 .

Step 5: Set $k:=k+1$. Solve problem (17) for finding an optimal solution to maximize DM2's membership function $\mu_{1}\left(p_{1}\left(\boldsymbol{x}_{1}, \boldsymbol{x}_{2}\right)\right)$ under the condition that DM1's membership function $\mu_{1}\left(p_{1}\left(\boldsymbol{x}_{1}, \boldsymbol{x}_{2}\right)\right)$ is larger than or equal to the minimal satisfactory level $\hat{\delta}$. For the optimal solution $\left(\boldsymbol{x}_{1}^{k}, \boldsymbol{x}_{2}^{k}\right)$ to (17), calculate $p_{l}^{k}=p_{l}\left(\boldsymbol{x}_{1}^{k}, \boldsymbol{x}_{2}^{k}\right), \mu_{l}\left(p_{l}^{k}\right), l=1$, 2, and $\Delta^{k}=$ $\mu_{2}\left(p_{2}^{k}\right) / \mu_{1}\left(p_{1}^{k}\right)$ and go to step 6 .

Step 6: If the current solution $\left(\boldsymbol{x}_{1}^{k}, \boldsymbol{x}_{2}^{k}\right)$ satisfies the termination conditions and DM1 accepts it, then the procedure stops and the current solution becomes a satisfactory solution. Otherwise, ask DM1 to update the minimal satisfactory level $\widehat{\delta}$, and go to step 5 .

It should be noted that all problems (8), (11), (17), (20) and (21) in the interactive fuzzy programming algorithm can be solved by either the simplex method of linear programming or the combined use of the bisection method and the phase one of the simplex method.

\section{Numerical example}

To demonstrate the feasibility and efficiency of the proposed method, consider the stochastic two-level linear programming problem formulated as:

$$
\left.\begin{array}{cl}
\operatorname{minimize} & \bar{z}_{1}\left(\boldsymbol{x}_{1}, \boldsymbol{x}_{2}\right)=\left(\boldsymbol{c}_{11}^{1}+\bar{t}_{1} \boldsymbol{c}_{11}^{2}\right) \boldsymbol{x}_{1}+\left(\boldsymbol{c}_{12}^{1}+\bar{t}_{1} \boldsymbol{c}_{12}^{2}\right) \boldsymbol{x}_{2}+\left(\alpha_{1}^{1}+\bar{t}_{1} \alpha_{1}^{2}\right) \\
\text { for DM1 } & \left.\bar{t}_{2}\right) \\
\text { minimize } & \bar{z}_{2}\left(\boldsymbol{x}_{1}, \boldsymbol{x}_{2}\right)=\left(\boldsymbol{c}_{21}^{1}+\bar{t}_{2} \boldsymbol{c}_{21}^{2}\right) \boldsymbol{x}_{1}+\left(\boldsymbol{c}_{22}^{1}+\bar{t}_{2} \boldsymbol{c}_{22}^{2}\right) \boldsymbol{x}_{2}+\left(\alpha_{2}^{1}+\bar{t}_{2} \alpha_{2}^{2}\right) \\
\text { for DM2 } & a_{i 1} \boldsymbol{x}_{1}+a_{i 2} \boldsymbol{x}_{2} \leq \bar{b}_{i}, i=1,2, \ldots, 7 \\
\text { subject to } & \boldsymbol{x}_{1}=\left(x_{11}, x_{12}, x_{13}, x_{14}, x_{15}\right)^{T} \geq \mathbf{0}, \\
& \boldsymbol{x}_{2}=\left(x_{21}, x_{22}, x_{23}, x_{24}, x_{25}\right)^{T} \geq \mathbf{0},
\end{array}\right\}
$$

where $\bar{t}_{1}$ and $\bar{t}_{2}$ are Gaussian random variables $N(4,22)$ and $N(3,32)$, and right side coefficients $\bar{b}_{i}, i=1,2, \ldots, 7$ are also Gaussian random variables $N(164,302), N(-190,202), N(-184,152), N(99,222), N(-150,172), N(154,352), N(142$, 
422). Here $N\left(p, q^{2}\right)$ stands for a Gaussian random variable with mean $p$ and variance $q^{2}$. Coefficient values of objective functions and constraints are respectively shown in Table 1 and 2.

Table 1. Coefficient values of objective functions.

\begin{tabular}{|c|c|c|c|c|c|c|c|c|c|c|c|c|}
\hline$\left(c_{11}^{1}, c_{12}^{1}\right)$ & 19 & 48 & 21 & 10 & 18 & 35 & 46 & 11 & 24 & 33 & $\alpha_{1}^{1}$ & -18 \\
\hline$\left(c_{11}^{2}, c_{12}^{2}\right)$ & 3 & 2 & 2 & 1 & 4 & 3 & 1 & 2 & 4 & 2 & $\alpha_{1}^{2}$ & 5 \\
\hline$\left(c_{21}^{1}, c_{22}^{1}\right)$ & 12 & -46 & -23 & -38 & -33 & -48 & 12 & 8 & 19 & 20 & $\alpha_{2}^{1}$ & -27 \\
\hline$\left(c_{21}^{2}, c_{22}^{2}\right)$ & 1 & 2 & 4 & 2 & 2 & 1 & 2 & 1 & 2 & 1 & $\alpha_{2}^{2}$ & 6 \\
\hline
\end{tabular}

Table 2. Coefficient values of constraints.

\begin{tabular}{|l|l|l|l|l|l|l|l|l|l|l|}
\hline$\left(a_{11}, a_{12}\right)$ & 12 & -2 & 4 & -7 & 13 & -1 & -6 & 6 & 11 & -8 \\
\hline$\left(a_{21}, a_{22}\right)$ & -2 & 5 & 3 & 16 & 6 & -12 & 12 & 4 & -7 & -10 \\
\hline$\left(a_{31}, a_{32}\right)$ & 3 & -16 & -4 & -8 & -8 & 2 & -12 & -12 & 4 & -3 \\
\hline$\left(a_{41}, a_{42}\right)$ & -11 & 6 & -5 & 9 & -1 & 8 & -4 & 6 & -9 & 6 \\
\hline$\left(a_{51}, a_{52}\right)$ & -4 & 7 & -6 & -5 & 13 & 6 & -2 & -5 & 14 & -6 \\
\hline$\left(a_{61}, a_{62}\right)$ & 5 & -3 & 14 & -3 & -9 & -7 & 4 & -4 & -5 & 9 \\
\hline$\left(a_{71}, a_{72}\right)$ & -3 & -4 & -6 & 9 & 6 & 18 & 11 & -9 & -4 & 7 \\
\hline
\end{tabular}

In step 1 of the interactive fuzzy programming, DM1 specifies satisficing levels $\beta_{i}, i=1,2, \ldots, 7$ as:

$$
\left(\beta_{1}, \beta_{2}, \beta_{3}, \beta_{4}, \beta_{5}, \beta_{6}, \beta_{7}\right)^{T}=(0.85,0.95,0.80,0.90,0.85,0.80,0.90)^{T} .
$$

For the specified satisficing levels $\beta_{i}, i=1,2, \ldots, 7$, in step 2, minimal values $z_{l, \text { min }}^{E}$ and maximal values $z_{l, \text { max }}^{E}$ of objective functions $\mathrm{E}\left\{\bar{z}_{l}\left(\boldsymbol{x}_{1}, \boldsymbol{x}_{2}\right)\right\}$ under the chance constraints are calculated as $z_{1 \text {, min }}^{E}=1819.513, z_{2 \text {,min }}^{E}=286.583$, $z_{1, \text { max }}^{E}=2307.626$ and $z_{2, \text { max }}^{E}=758.279$. By considering these values, the DMs subjectively specifies permissible levels as $h_{1}=2150.0$ and $h_{2}=450.0$.

In step 3, maximal values $p_{l, \max }$ of $p_{l}\left(\boldsymbol{x}_{1}, \boldsymbol{x}_{2}\right)$ are calculated as:

$$
p_{1, \text { max }}=p_{1}\left(\boldsymbol{x}_{1, \text { max }}^{1}, \boldsymbol{x}_{2, \text { max }}^{1}\right)=0.880, p_{2, \text { max }}=p_{2}\left(\boldsymbol{x}_{1, \text { max }}^{2}, \boldsymbol{x}_{2, \text { max }}^{2}\right)=0.783
$$

Assume that the DMs identify the linear membership function (7) whose parameter values are determined by the Zimmermann method ${ }^{[54]}$. Then, the parameter values $p_{l, 1}$ and $p_{l, 0}, l=1,2$ characterizing membership functions $\mu_{l}(\cdot)$ are becomes:

$$
\begin{aligned}
& p_{1,1}=p_{1}\left(\boldsymbol{x}_{1, \text { max }}^{1}, \boldsymbol{x}_{2, \text { max }}^{1}\right)=0.880, \\
& p_{1,0}=p_{1}\left(\boldsymbol{x}_{1, \text { max }}^{2}, \boldsymbol{x}_{2, \text { max }}^{2}\right)=0.598, \\
& p_{2,1}=p_{2}\left(\boldsymbol{x}_{1, \text { max }}^{2}, \boldsymbol{x}_{2, \text { max }}^{2}\right)=0.783, \\
& p_{2,0}=p_{2}\left(\boldsymbol{x}_{1, \text { max }}^{1}, \boldsymbol{x}_{2, \text { max }}^{1}\right)=0.060 .
\end{aligned}
$$

In step 4, let $k:=1$ and the maximin problem is solved. The obtained result is shown at the column labeled " 1 st" in Table 3. For the obtained optimal solution $\left(\boldsymbol{x}_{1}^{1}, \boldsymbol{x}_{2}^{1}\right)$ to the maximin problem, corresponding membership function values are calculated as $\mu_{1}\left(p_{1}\left(\boldsymbol{x}_{1}^{1}, \boldsymbol{x}_{2}^{1}\right)\right)=0.551$ and $\mu_{2}\left(p_{2}\left(\boldsymbol{x}_{1}^{1}, \boldsymbol{x}_{2}^{1}\right)\right)=0.551$. Then, the ratio of satisfactory degrees $\Delta^{1}$ is equal to 1.000. Since DM1 is not satisfied with this solution, DM1 sets the minimal satisfactory level $\widehat{\delta} \in(0,1)$ for $\mu_{1}\left(p_{1}\left(\boldsymbol{x}_{1}, \boldsymbol{x}_{2}\right)\right)$ 
to 0.600 so that $\mu_{1}\left(p_{1}\left(\boldsymbol{x}_{1}, \boldsymbol{x}_{2}\right)\right)$ will be improved from its current value 0.551 . Furthermore, the upper bound and the lower bound of the ratio of satisfactory degrees $\Delta$ are set as $\Delta_{\max }=0.700$ and $\Delta_{\min }=0.600$.

Table 3. Interaction process.

\begin{tabular}{lllll}
\hline Interaction & 1st & 2nd & 3rd & 4th \\
\hline$\widehat{\delta}$ & & 0.600 & 0.700 & 0.650 \\
$\boldsymbol{x}_{11}^{k}$ & 15.368 & 15.066 & 14.423 & 14.749 \\
$\boldsymbol{x}_{12}^{k}$ & 2.162 & 1.960 & 1.532 & 1.750 \\
$\boldsymbol{x}_{13}^{k}$ & 0.000 & 0.000 & 0.000 & 0.000 \\
$\boldsymbol{x}_{14}^{k}$ & 0.000 & 0.000 & 0.000 & 0.000 \\
$\boldsymbol{x}_{15}^{k}$ & 0.000 & 0.000 & 0.000 & 0.000 \\
$\boldsymbol{x}_{21}^{k}$ & 6.033 & 5.784 & 5.255 & 5.524 \\
$\boldsymbol{x}_{22}^{k}$ & 0.118 & 0.108 & 0.086 & 0.097 \\
$\boldsymbol{x}_{23}^{k}$ & 14.276 & 14.489 & 14.953 & 14.707 \\
$\boldsymbol{x}_{24}^{k}$ & 1.516 & 1.775 & 2.325 & 2.046 \\
$\boldsymbol{x}_{25}^{k}$ & 17.848 & 17.997 & 18.315 & 18.153 \\
$p_{1}\left(\boldsymbol{x}_{1}^{k}, \boldsymbol{x}_{2}^{k}\right)$ & 0.734 & 0.767 & 0.796 & 0.781 \\
$p_{2}\left(\boldsymbol{x}_{1}^{k}, \boldsymbol{x}_{2}^{k}\right)$ & 0.458 & 0.406 & 0.301 & 0.353 \\
$\mu_{1}\left(p_{1}\left(\boldsymbol{x}_{1}^{k}, \boldsymbol{x}_{2}^{k}\right)\right)$ & 0.551 & 0.600 & 0.700 & 0.650 \\
$\mu_{2}\left(p_{2}\left(\boldsymbol{x}_{1}^{k}, \boldsymbol{x}_{2}^{k}\right)\right)$ & 0.551 & & 0.478 & 0.405 \\
$\Delta^{k}$ & 1.000 & 0.797 & 0.475 & 0.623 \\
\hline
\end{tabular}

In step 5, let $k:=2$ and (17) for $\widehat{\delta}=0.600$ is solved. For the obtained optimal solution $\left(\boldsymbol{x}_{1}^{2}, \boldsymbol{x}_{2}^{2}\right)$ to $(17), \mu_{1}\left(p_{1}\left(\boldsymbol{x}_{1}^{2}, \boldsymbol{x}_{2}^{2}\right)\right)=$ 0.600 and $\mu_{2}\left(p_{2}\left(\boldsymbol{x}_{1}^{2}, \boldsymbol{x}_{2}^{2}\right)\right)=0.478$, and $\Delta^{2}=0.797$, shown at the column labeled " 2 nd" in Table 3 .

In step 6, DM1 is asked whether he is satisfied with the obtained solution. Since the ratio of satisfactory degrees $\Delta^{2}$ exceeds $\Delta_{\max }=0.700$, the second condition of termination of the interactive process is not fulfilled. Suppose that DM1 feels that $\mu_{1}\left(p_{1}\left(\boldsymbol{x}_{1}, \boldsymbol{x}_{2}\right)\right)$ should be considerably better than $\mu_{2}\left(p_{2}\left(\boldsymbol{x}_{1}, \boldsymbol{x}_{2}\right)\right)$, and DM1 updates the minimal satisfactory level $\widehat{\delta}$ from 0.600 to 0.700 in order to improve $\mu_{1}\left(p_{1}\left(\boldsymbol{x}_{1}, \boldsymbol{x}_{2}\right)\right)$. Consequently, in step 5, let $k:=3$ and (17) for $\widehat{\delta}=0.700$ is solved. The obtained result is shown at the column labeled " 3 rd" in Table 3 . For the obtained optimal solution $\left(\boldsymbol{x}_{1}^{3}, \boldsymbol{x}_{2}^{3}\right)$ to (17), $\mu_{1}\left(p_{1}\left(\boldsymbol{x}_{1}^{3}, \boldsymbol{x}_{2}^{3}\right)\right)=0.700, \mu_{2}\left(p_{2}\left(\boldsymbol{x}_{1}^{3}, \boldsymbol{x}_{2}^{3}\right)\right)=0.333$ and $\Delta^{3}=0.475$.

In step 6 , since the ratio of satisfactory degrees $\Delta^{3}$ is less than $\Delta_{\min }=0.600$, the second condition of termination of the interactive process is not fulfilled. Hence, he updates the minimal satisfactory level $\delta$ from 0.700 to 0.650 for improving $\mu_{2}\left(p_{2}\left(\boldsymbol{x}_{1}, \boldsymbol{x}_{2}\right)\right)$ at the sacrifice of $\mu_{1}\left(p_{1}\left(\boldsymbol{x}_{1}, \boldsymbol{x}_{2}\right)\right)$. As a result, in step 5, let $k:=4$ and (17) for $\delta=0.650$ is solved. For the obtained optimal solution $\left(\boldsymbol{x}_{1}^{4}, \boldsymbol{x}_{2}^{4}\right)$ to (17), corresponding membership function values are calculated as $\mu_{1}\left(p_{1}\left(\boldsymbol{x}_{1}^{4}, \boldsymbol{x}_{2}^{4}\right)\right)=$ $0.650, \mu_{2}\left(p_{2}\left(x_{1}^{4}, x_{2}^{4}\right)\right)=0.405$ as shown at the column labeled " 4 th" in Table 3 . Then, the ratio of satisfactory degrees $\Delta^{4}$ is equal to 0.623 .

In step 6, since the current solution satisfies all termination conditions of the interactive process and DM1 is satisfied with the current solution, the satisfactory solution is obtained and the interaction procedure is terminated.

It must be observed here that the DMs may become possible to specify permissible levels for the objective functions and change the minimal satisfactory level interactively due to learning or improved understanding during the solution process. 


\section{Conclusions}

In this paper, we focused on stochastic two-level linear programming problems with random variable coefficients in both objective functions and constraints. Through the use of the probability maximization model in chance constrained programming, the stochastic two-level linear programming problems are transformed into deterministic linear programming ones under some appropriate assumptions for distribution functions. Taking into account vagueness of judgments of the DMs, interactive fuzzy programming has been proposed. In the proposed interactive method, after determining the fuzzy goals of the DMs at both levels, a satisfactory solution is derived efficiently by updating the satisfactory degree of the DM at the upper level with considerations of overall satisfactory balance among both levels. It is significant to note here that the transformed deterministic problems to derive an overall satisfactory solution can be easily solved through the combined use of the bisection method and the phase one of the simplex method. An illustrative numerical example was provided to demonstrate the feasibility of the proposed method. Although the DMs are required to specify permissible levels for the objective functions and change the minimal satisfactory level interactively, such a task seems to become possible for the DMs due to learning or improved understanding during the solution process.

However, applications must be carried out in corporation with a person actually involved in decision making. From such experiences the proposed method must be revised. Extensions to other stochastic programming models will be considered elsewhere. Especially, it would be interesting to construct models optimizing not only the probability levels but also other parameters such as aspiration levels.

Also extensions to two-level linear programming problems involving not only random variable coefficients but also fuzzy random or random fuzzy variable coefficients will be required in the near future.

\section{References}

[1] W.F. Bialas and M.H. Karwan, Two-level linear programming, Management Science. 1984; 30(8): 1004-1020. http://dx.doi.org/10.1287/mnsc.30.8.1004

[2] Nishizaki and M. Sakawa, Computational methods through genetic algorithms for obtaining Stackelberg solutions to two-level mixed zero-one programming problems, Cybernetics and Systems: An International Journal. 2000; 31(2): $203-221$. http://dx.doi.org/10.1080/019697200124892

[3] K. Shimizu, Y. Ishizuka and J.F. Bard, Nondifferentiable and Two-Level Mathematical Programming, Kluwer Academic Publishers, Boston, 1997. http://dx.doi.org/10.1007/978-1-4615-6305-1

[4] M. Simaan and J.B. Cruz, Jr., On the Stackelberg strategy in nonzero-sum games, Journal of Optimization Theory and Applications. 1973; 11(5): 533-555. http://dx.doi.org/10.1007/BF00935665

[5] Y.J. Lai, Hierarchical optimization: a satisfactory solution, Fuzzy Sets and Systems. 1996; 77(3): 321-335. http://dx.doi.org/10.1016/0165-0114(95)00086-0

[6] H.S. Shih, Y.J. Lai and E.S. Lee, Fuzzy approach for multi-level programming problems, Computers and Operations Research. 1996; 23(1): 73-91. http://dx.doi.org/10.1016/0305-0548(95)00007-9

[7] M. Sakawa, I. Nishizaki and Y. Uemura, Interactive fuzzy programming for multi-level linear programming problems, Computers \& Mathematics with Applications. 1998; 36(2): 71-86. http://dx.doi.org/10.1016/S0898-1221(98)00118-7

[8] M. Sakawa, I. Nishizaki and Y. Uemura, Interactive fuzzy programming for two-level linear fractional programming problems with fuzzy parameters, Fuzzy Sets and Systems. 2000; 115: 93-103. http://dx.doi.org/10.1016/S0898-1221(98)00118-7

[9] M.A. Abo-Sinna and I.A. Baky, Interactive balance space approach for solving multi-level multi-objective programming problems, Information Sciences. 2007; 177(16): 3397-3410. http://dx.doi.org/10.1016/j.ins.2007.02.005

[10] E.S. Lee, Fuzzy multiple level programming, Applied Mathematics and Computation. 2001; 120(1-3): 79-90.

[11] E. Roghanian, S.J. Sadjadi and M.B. Aryanezhad, A probabilistic bi-level linear multi-objective programming problem to supply chain planning, Applied Mathematics and Computation. 2007; 188(1): 786-800. http://dx.doi.org/10.1016/j.amc.2006.10.032

[12] M. Sakawa and I. Nishizaki, Interactive fuzzy programming for decentralized two-level linear programming problems, Fuzzy Sets and Systems. 2002; 125(3): 301-315. http://dx.doi.org/10.1016/S0165-0114(01)00042-2 
[13] M. Sakawa and I. Nishizaki, Interactive fuzzy programming for two-level nonconvex programming problems with fuzzy parameters through genetic algorithms, Fuzzy Sets and Systems. 2002; 127(2): 185-197. http://dx.doi.org/10.1016/S0165-0114(01)00134-8

[14] M. Sakawa and I. Nishizaki, Cooperative and Noncooperative Multi-Level Programming, Springer, New York, 2009.

[15] M. Sakawa, I. Nishizaki and H. Katagiri, Fuzzy Stochastic Multiobjective Programming, Springer, New York, 2011. http://dx.doi.org/10.1007/978-1-4419-8402-9

[16] M. Sakawa, I. Nishizaki and Y. Uemura, Interactive fuzzy programming for two-level linear and linear fractional production and assignment problems: a case study, European Journal of Operational Research. 2001; 135(1): 142-157. http://dx.doi.org/10.1016/S0377-2217(00)00309-X

[17] M. Sakawa, I. Nishizaki and Y. Uemura, A decentralized two-level transportation problem in a housing material manufacturerInteractive fuzzy programming approach, European Journal of Operational Research. 2002; 141(1): 167-185. http://dx.doi.org/10.1016/S0377-2217(01)00273-9

[18] S. Sinha, Fuzzy programming approach to multi-level programming problems, Fuzzy Sets and Systems. 2003 ; $136(2): 189-202$. http://dx.doi.org/10.1016/S0165-0114(02)00362-7

[19] J.R. Birge and F. Louveaux, Introduction to Stochastic Programming, Springer, London, 1997.

[20] I.M. Stancu-Minasian, Overview of different approaches for solving stochastic programming problems with multiple objective functions, R. Slowinski and J. Teghem (eds.): Stochastic Versus Fuzzy Approaches to Multiobjective Mathematical Programming under Uncertainty, Kulwer Academic Publishers, Dordrecht/Boston/London. 1990: 71-101.

[21] G.B. Dantzig, Linear programming under uncertainty, Management Science. 1995; 1(3-4): 197-206. http://dx.doi.org/10.1287/mnsc.1.3-4.197

[22] Charnes and W.W. Cooper, Chance constrained programming, Management Science. 1959; 6(1): 73-79. http://dx.doi.org/10.1287/mnsc.6.1.73

[23] I.M. Stancu-Minasian, Stochastic Programming with Multiple Objective Functions, D. Reidel Publishing Company, Dordrecht, 1984.

[24] J.-P. Leclercq, Stochastic programming: an interactive multicriteria approach, European Journal of Operational Research. 1982; 10: 33-41. http://dx.doi.org/10.1016/0377-2217(82)90129-1

[25] J. Teghem Jr., D. Dufrane, M. Thauvoye, and P. Kunsch, STRANGE: an interactive method for multi-objective linear programming under uncertainty, European Journal of Operational Research. 1986; 26: 65-82. http://dx.doi.org/10.1016/0377-2217(86)90160-8

[26] H. Rommelfanger, Fuzzy linear programming and applications, European Journal of Operational Research. 1996; $92(3): 512-527$. http://dx.doi.org/10.1016/0377-2217(95)00008-9

[27] M. Sakawa, Fuzzy Sets and Interactive Multiobjective Optimization, Plenum Press, New York, 1993.

[28] H.-J. Zimmermann, Fuzzy programming and linear programming with several objective functions, Fuzzy Sets and Systems. 1978; 1(1): 45-55. http://dx.doi.org/10.1016/0165-0114(78)90031-3

[29] Y.J. Lai and C.L. Hwang, Fuzzy Mathematical Programming, Springer-Verlag, Berlin, 1992. http://dx.doi.org/10.1007/978-3-642-48753-8

[30] M. K. Luhandjula, Multiple objective programming problems with possibilistic coefficients, Fuzzy Sets and Systems. 1987; 21: 135-145. http://dx.doi.org/10.1016/0165-0114(87)90159-X

[31] M. Sakawa, Large Scale Interactive Fuzzy Multiobjective Programming, Physica-Verlag, Heidelberg, 2000. http://dx.doi.org/10.1007/978-3-7908-1851-2

[32] M. Sakawa. Genetic Algorithms and Fuzzy Multiobjective Optimization, Kluwer Academic Publishers, Boston, 2001. PMid: 18244812

[33] M. Sakawa, H. Yano, and T. Yumine, An interactive fuzzy satisficing method for multiobjective linear-programming problems and its application, IEEE Transactions on Systems, Man, and Cybernetics. 1987; SMC-17: 654-661. http://dx.doi.org/10.1109/TSMC.1987.289356

[34] R. Slowinski (ed.), Fuzzy Sets in Decision Analysis, Operations Research and Statistics, Kluwer Academic Publishers, Dordrecht/Boston/London, 1998. http://dx.doi.org/10.1007/978-1-4615-5645-9

[35] R. Slowinski and J. Teghem (eds.), Stochastic Versus Fuzzy Approaches to Multiobjective Mathematical Programming under Uncertainty, Kluwer Academic Publishers, Dordrecht/Boston/London, 1990. http://dx.doi.org/10.1007/978-94-009-2111-5

[36] H.-J. Zimmermann, Fuzzy Sets, Decision-Making and Expert Systems, Boston: Kluwer Academic Publishers, 1987. http://dx.doi.org/10.1007/978-94-009-3249-4

[37] G.-Y. Wang, and Z. Qiao, Fuzzy programming with fuzzy random variable coefficients, Fuzzy Sets and Systems. 1993; 57(3): 295-311. http://dx.doi.org/10.1016/0165-0114(93)90025-D 
[38] B. Liu, and K. Iwamura, Chance constrained programming with fuzzy parameters, Fuzzy Sets and Systems. $1998 ; 94(2): 227-237$. http://dx.doi.org/10.1016/S0165-0114(96)00236-9

[39] S. Hulsurkar, M.P. Biswal and S.B. Sinha, Fuzzy programming approach to multi-objective stochastic linear programming problems, Fuzzy Sets and Systems. 1997; 88(2): 173-181. http://dx.doi.org/10.1016/S0165-0114(96)00056-5

[40] M. Sakawa and K. Kato, An interactive fuzzy satisficing method for multiobjective stochastic linear programming problems using chance constrained conditions, Journal of Multi-Criteria Decision Analysis. 2002; 11: 125-137. http://dx.doi.org/10.1016/S0165-0114(96)00056-5

[41] M. Sakawa, K. Kato and H. Katagiri, An interactive fuzzy satisficing method through a variance minimization model for multiobjective linear programming problems involving random variables. KES Journal. 2002; 2: 1222-1226.

[42] M. Sakawa, K. Kato and I. Nishizaki, An interactive fuzzy satisficing method for multiobjective stochastic linear programming problems through an expectation model. European Journal of Operational Research. 2003; 145(3): 665-672. http://dx.doi.org/10.1016/S0377-2217(02)00150-9

[43] M. Sakawa and H. Yano, Interactive fuzzy satisficing method using augmented minimax problems and its application to environmental systems, IEEE Transactions on Systems, Man and Cybernetics. 1985; SMC-15: 720-729. http://dx.doi.org/10.1109/TSMC.1985.6313455

[44] M. Sakawa and H. Yano, Interactive decision making for multiobjective nonlinear programming problems with fuzzy parameters, Fuzzy Sets and Systems. 1989; 29: 315-326. http://dx.doi.org/10.1016/0165-0114(89)90043-2

[45] M. Sakawa and H. Yano, An interactive fuzzy satisficing method for generalized multiobjective linear programming problems with fuzzy parameters, Fuzzy Sets and Systems. 1990; 35: 125-142. http://dx.doi.org/10.1016/0165-0114(90)90188-C

[46] P. Guo, and G. H. Huang, Two-stage fuzzy chance-constrained programming -application to water resources management under dual uncertainties, Stochastic Environmental Research and Risk Assessment. 2009; 23: 2349-359. http://dx.doi.org/10.1007/s00477-008-0221-y

[47] Y.P. Li, and G.H. Huang, Fuzzy stochastic based violation analysis method for planning water resources management systems with uncertain information, Information Sciences. 2009; 179: 4261-4276. http://dx.doi.org/10.1016/j.ins.2009.09.001

[48] Y.P. Li, G.H. Huang, Y.F. Huang, H.D. Zhou, A multistage fuzzy-stochastic programming model for supporting sustainable water resources allocation and management, Environmental Modelling \& Software. 2009; 24: 786-797. http://dx.doi.org/10.1016/j.envsoft.2008.11.008

[49] H. Katagiri, M. Sakawa, K. Kato and I. Nishizaki, Interactive multiobjective fuzzy random linear programming: Maximization of possibility and probability, European Journal of Operational Research. 2008; 188(2): 530-539. http://dx.doi.org/10.1016/j.ejor.2007.02.050

[50] H. Katagiri and M. Sakawa, Interactive multiobjective fuzzy random programming through the level-set based probability model, Information Sciences. 2011; 181(9): 1641-1650. http://dx.doi.org/10.1016/j.ins.2011.01.003

[51] Charnes and W.W. Cooper, Deterministic equivalents for optimizing and satisficing under chance constraints, Operations Research. 1963; 11(1): 18-39. http://dx.doi.org/10.1287/opre.11.1.18

[52] S. Kataoka, A stochastic programming model, Econometorica. 1963; 31(1-2): 181-196. http://dx.doi.org/10.2307/1910956

[53] R.E. Bellman and L.A. Zadeh, Decision making in a fuzzy environment, Management Science. 1970; 17(4): 141-164. http://dx.doi.org/10.1287/mnsc.17.4.B141

[54] Charnes and W.W. Cooper, Programming with linear fractional functions, Naval Research Logistics Quaterly. 1962; 9(3-4): 181-186. 